\title{
ASPECTOS SOCIALES PARA FOMENTAR LA PERMANENCIA EN LAS INSTITUCIONES DE EDUCACIÓN SUPERIOR EN AMBIENTES VIRTUALES DE APRENDIZAJE
}

\section{SOCIAL ASPECTS TO PROMOTE PERMANENCE IN INSTITUTIONS OF HIGHER EDUCATION IN VIRTUAL LEARNING ENVIRONMENTS}

\section{Autor 1}

Guillermo Giraldo Vargas

Administrador de empresas, especialista en gestión de costos, magister en administración de organizaciones, y tecnólogo en gestión agropecuaria. Docente ocasional de la UNAD, y perteneciente al grupo de investigación CANANGUCHALES (Código COL0060156), y al semillero de investigación SIDESGOP. ORCID: 0000-0003-2695-4745

Correo electrónico: guillermo.giraldo@unad.edu.co

\section{Autor 2}

Henry Hincapié López

Especialista en Proceso de calidad, Ingeniero industrial. Docente ocasional de la UNAD, y perteneciente al grupo de investigación CANANGUCHALES (Código COL0060156), y al semillero de investigación SIDESGOP. ORCID: 0000-0002-5242-4345

Correo electrónico: henry.hincapie@unad.edu.co

\section{Autor 3}

Nelson Augusto Serna Porras

Maestrando en Administración de Organizaciones, Administrador de Empresas. Docente ocasional de la UNAD, y perteneciente al grupo de investigación CANANGUCHALES (Código COL0060156), y al semillero de investigación SIDESGOP.

ORCID: 0000-0002-4575-4645

Correo electrónico: nelson.serna@unad.edu.co

\section{RESUMEN}

Las acciones realizadas para propiciar la permanencia de los estudiantes en los programas de educación superior son muy variadas y van desde políticas definidas por el ministerio de educación nacional (MEN), hasta estrategias diseñadas por las instituciones de educación superior (IES). Lo que ocurre es que estas acciones en su mayoría se han orientado a facilitarle a los 
estudiantes, la mejor manera de superar la incidencia que tienen los factores económicos en la permanencia de sus estudios, y muy pocas se enfocan en aspectos considerados menores, pero que pueden llegar a contribuir en permanencia de los estudiantes, al valorar los talentos o hobbies con que cuentan los estudiantes. Por lo tanto, este documento de trabajo muestra la importancia que algunos factores sociales de la vida universitaria pueden llegar a tener en la permanencia de los estudiantes en sus programas académicos matriculados, lo cual no se aleja de lo que deben hacer las instituciones. Este trabajo es fruto de una investigación no experimental, descriptiva y transversal que permitió describir los factores que más incidencia tienen en la decisión de abandonar o permanecer en un programa matriculado de la escuela ECACEN, además de recolectar información primaria de estudiantes catalogados como antiguos, y una revisión detallada otras investigaciones acerca del fenómeno de la deserción, sin embargo es necesario señalar que para el caso de este documento de trabajo se seleccionan solo las preguntas relacionadas con los talentos que poseen los estudiantes y las que indagan acerca de la motivación para permanecer en los programas. De esta manera, se encuentra que los talentos o hobbies que poseen los estudiantes pueden ser utilizados para propiciar un mejor ambiente universitario y contribuir a la permanencia en sus programas, además de verificar que el desarrollo de estos talentos, que también ayudan a la realización del ser humano, puede ser impulsado por las instituciones de educación superior.

Palabras clave: aspectos sociales, permanencia, talentos, hobby, deserción, bienestar.

\section{ABSTRACT}

The actions carried out to promote the permanence of students in higher education programs are very varied and range from policies defined by the national ministry of education (MEN), to strategies designed by higher education institutions (HEIs). What happens is that the majority of these actions have been aimed at providing students with the best way to overcome the impact that economic factors have on the permanence of their studies, and very few focus on aspects considered minor, but that they can contribute to the permanence of the students, by valuing the talents or hobbies that the students have. Therefore, this working document shows the importance that some social factors of university life may have in the permanence of students in their enrolled academic programs, which is not far from what institutions must do. 
This work is the result of a non-experimental, descriptive and cross-sectional investigation that allowed describing the factors that have the greatest incidence in the decision to leave or stay in an enrolled program of the ECACEN school, in addition to collecting primary information from students classified as former, and A detailed review other investigations about the phenomenon of the desertion, but nevertheless it is necessary to point out that in the case of this work document, only the questions related to the talents that the students possess and those that inquire about the motivation to remain are selected in the programs. In this way, it is found that the talents or hobbies that the students possess can be used to promote a better university environment and contribute to the permanence in their programs, in addition to verifying that the development of these talents, which also help to carry out the human being, can be driven by higher education institutions.

Keywords: Social Aspects, Permanence, Talents, Hobby, Desertion, Wellness.

\section{INTRODUCCIÓN}

El presente documento de trabajo es producto de una investigación mayor culminada en el año 2020, donde se diseñó un modelo de gestión del conocimiento que permita la permanecía de los estudiantes matriculados en los programas de la escuela ECACEN de la UNAD. Para diseñar dicho modelo se recolecta información primaria de estudiantes catalogados como antiguos, a través de la aplicación de un cuestionario donde se indaga acerca de los factores que pueden tener incidencia con su permanencia o abandono de su programa académico matriculado, pero además se incluyen unas preguntas que buscan conocer la motivación de los estudiantes y los talentos o hobbies que poseen. Es así como a partir de estas últimas preguntas se construye este documento de trabajo, que pretende mostrar la importancia que puede llegar a tener para la universidad, el aprovechamiento de los talentos que poseen los estudiantes, apoyarlos en su desarrollo y de esta manera contribuir al bienestar institucional y a la permanencia de los estudiantes. Por lo tanto, el lector al inicio de este documento encuentra los factores que tienen una gran incidencia en el abandono de los estudios por parte de los estudiantes. Dichos factores son muy similares a los identificados por otros investigadores y que coinciden en ser económicos, académicos, institucionales, familiares e individuales.

Luego se describen los factores que motivan a los estudiantes a continuar sus estudios, entre los cuales se encuentran el rendimiento académico, una buena relación con la universidad y con sus compañeros de estudio, así como las razones que los impulsan a culminar sus estudios satisfactoriamente. 
Para culminar el documento, señalando la importancia que pueden llegar a tener el aprovechamiento de los talentos o hobbies que poseen los estudiantes, por parte de las instituciones de educación superior, con la finalidad de contribuir al bienestar del estudiante y propiciar la permanencia. Para lograr lo anterior se consultan varios autores y se buscan puntos coincidentes entre lo señalado por los estudiantes en la encuesta, los resultados de investigaciones de otros autores, y la interpretación del investigador principal.

Para la educación superior mediada a través de la virtualidad, donde se hace necesario crear comunidad académica más sólida, ya sea a través de espacios virtuales o presenciales, puede llegar a ser muy valioso cultivar los talentos de sus estudiantes.

\section{METODOLOGÍA}

El presente documento es fruto de una investigación no experimental, "debido a que no manipula ninguna de las variables presentes" (Lerma, 2012). Además, corresponde al tipo descriptivo, que permite conocer los factores y aspectos sociales que inciden en el abandono o permanencia de los estudiantes en los programas matriculados, y por recolectar información primaria en un momento único, se cataloga como transversal.

\section{Población.}

La población objeto de estudio para esta investigación, corresponde a los estudiantes catalogados como antiguos, matriculados en los programas académicos de la escuela ECACEN de la zona occidente en el periodo 16-01 de 2019. Para ese momento se tiene una población de 862 estudiantes.

\section{Muestra.}

La muestra representativa de la población es de un tamaño de 266 elementos muéstrales. Dicha muestra se estratifica con el criterio de centros (CEAD o CCAV) pertenecientes a la zona occidente, con la finalidad de aplicar de una manera más equitativa el instrumento de recolección de información. Nota: El instrumento fue diligenciado por 276 estudiantes, es decir 10 estudiantes más.

\section{Unidad de análisis.}

La unidad de análisis de la cual se obtiene la información primaria corresponde a estudiantes matriculados en los programas de la escuela ECACEN en la zona occidente de la UNAD, catalogados como antiguos, 
condición que se cumple al superar el estudiante el primer periodo académico.

\section{DISCUSIÓN Y RESULTADOS}

Las respuestas brindadas por los estudiantes en el cuestionario aplicado, como instrumento de recolección de información primaria, para la construcción de un modelo de gestión del conocimiento que permita la retención de los estudiantes matriculados en los programa de la ECACEN, permite reconocer que las dificultades económicas que se le presenten a los estudiantes, pueden llegar a incidir en su permanencia en el programa matriculado, lo cual se valida con unos porcentajes del $37 \%$ de incidencia alta, y un $38 \%$ de incidencia alta, que representa tres cuartas partes de los estudiantes encuestados (Tabla 1 ).

Tabla 1

Incidencia del factor económico en la permanencia

\begin{tabular}{ccc}
\hline Valoración & F-Absoluta & F-Relativa \\
\hline Alta & 103 & $37 \%$ \\
Media & 106 & $38 \%$ \\
Baja & 41 & $15 \%$ \\
Ninguna incidencia & 26 & $9 \%$ \\
Total & 276 & $100 \%$ \\
\hline
\end{tabular}

También este factor ratifica su importancia, al encontrar que el $67 \%$ de los estudiantes financian sus estudios con recursos provenientes de una relación laboral, y un $18 \%$ con recursos familiares. Lo anterior muestra lo vulnerable que es la permanencia estudiantil ante las condiciones económicas favorables (Tabla 2).

Fuente: elaboración propia

Tabla 2 Recursos que financian los estudios

\begin{tabular}{ccc}
\hline Origen del recurso & $\begin{array}{c}\text { F- } \\
\text { Absoluta }\end{array}$ & $\begin{array}{c}\text { F- } \\
\text { Relativa }\end{array}$ \\
\hline $\begin{array}{c}\text { Recursos familiares } \\
\text { Recursos provenientes de una relación laboral }\end{array}$ & 184 & $67 \%$ \\
$\begin{array}{c}\text { Préstamos bancarios o de entidades de fomento } \\
\text { como el ICETEX }\end{array}$ & 51 & $18 \%$ \\
$\begin{array}{c}\text { Programas de benérios a la educación como becas } \\
\text { de estudio } \\
\text { Total }\end{array}$ & 19 & $7 \%$ \\
& 22 & $8 \%$ \\
& 276 & $100 \%$ \\
\hline
\end{tabular}


Fuente: elaboración propia

Pero contrario a los dos resultados anteriores, se encuentra que el rendimiento académico de los estudiantes en un $22 \%$ es sobresaliente, un $59 \%$ alto, y tan solo un $18 \%$ consideran su rendimiento como regular y bajo, que a su vez repercute en motivación para continuar sus estudios, debido a que el $70 \%$ se encuentra bastante motivado para continuar sus estudios, y también un $68 \%$ señala estar $100 \%$ capaz de culminar el programa que tienen matriculado, por razones como: haber obtenido aprendizajes significativos, por ser estudioso, porque la carrera es el arma para su futuro, porque le encanta la carrera, le falta poco para terminar la carrera, porque se sienten agradecidos con sus tutores, y por ser responsables y dedicados con sus estudios (Tablas 3 y 4 ).

Tabla 3

Rendimiento académico

\begin{tabular}{ccc}
\hline Valoración & F-Absoluta & F-Relativa \\
\hline Alto & 164 & $59 \%$ \\
Bajo & 3 & $1 \%$ \\
Muy bajo & 1 & $0 \%$ \\
Regular & 46 & $17 \%$ \\
Sobresaliente & 62 & $22 \%$ \\
Total & 276 & $100 \%$ \\
\hline
\end{tabular}

Fuente: elaboración propia

Además, como objetivo central de este documento de trabajo que busca identificar aspectos o factores sociales que permitan lograr la permanencia en estudiantes de educación superior mediados a través de la virtualidad, más precisamente en la UNAD, se encuentra que el $37 \%$ de los estudiantes encuestados, están totalmente de acuerdo en que una buena relación con los compañeros de estudio es determinante para continuar en la universidad, y un $31 \%$ también están de acuerdo (Tabla 5).

Tabla 4

Motivación de acuerdo con el rendimiento académico

\begin{tabular}{ccc}
\hline Valoración & F-Absoluta & F-Relativa \\
\hline Lo ha motivado bastante para continuar estudiando. & 192 & $70 \%$ \\
Lo ha motivado en algo para continuar estudiando & 71 & $25,30 \%$ \\
Lo ha motivado muy poco para continuar estudiando. & 13 & $4,70 \%$ \\
\hline
\end{tabular}


Fuente: elaboración propia

Tabla 5

Relación con los compañeros de estudio

\begin{tabular}{ccc}
\hline ítem & F-Absoluta & F-Relativa \\
\hline Estoy de acuerdo. & 85 & $31 \%$ \\
Estoy en desacuerdo. & 11 & $4 \%$ \\
Estoy medianamente de acuerdo. & 49 & $18 \%$ \\
Estoy totalmente de acuerdo. & 102 & $36 \%$ \\
No estoy muy de acuerdo. & 29 & $11 \%$ \\
Total & 276 & $100 \%$ \\
\hline
\end{tabular}

Fuente: elaboración propia

En este sentido Gómez \& Vinasco (2020), recogiendo opiniones de los estudiantes respecto a la importancia de la relación con sus compañeros de estudio, comparten la siguiente observación de un estudiante:

Cuando nos sentábamos a trabajar con mis amigos, cada uno de nosotros traía elementos distintos a nuestros grupos de estudio: las notas, las ideas y las diferentes formas de entender un mismo concepto; todo eso ayudaba a aprender mejor. Ahí caí en cuenta de que uno no solo aprende de sus profesores: también es posible aprender de pares y colegas. (Gómez \& Vinasco, 2020).

Sumado a lo anterior, al indagar a los estudiantes acerca de algún talento o hobbie que les gustaría practicar en la universidad, las respuestas son variadas y van desde los deportes, la danza, literatura y el cine. Pero además los estudiantes consideran que la universidad puede apoyar estos talentos a través de talleres, cursos, grupos de apoyo y equipos deportivos. Más sin embargo se debe tener presente que la universidad oferta programas profesionales como son: Artes visuales, gestión deportiva y música, además de tener en muchos de sus programas académicos, cursos complementarios como son teatro, guitarra, preparación física y danza (Tabla 6).

Tabla 6

Talentos o hobbies

\begin{tabular}{ccc}
\hline Talentos o hobbies & F-Absoluta & F-Relativa \\
\hline Cine & 14 & $5 \%$ \\
Danza & 29 & $11 \%$ \\
\hline
\end{tabular}




$\begin{array}{ccc}\text { Deportes } & 92 & 33 \% \\ \text { Literatura } & 31 & 11 \% \\ \text { Ninguno } & 79 & 29 \% \\ \text { Otro } & 31 & 11 \% \\ \text { Total } & 276 & 100 \%\end{array}$

Fuente: elaboración propia

Ahora bien, la importancia o relevancia que pueden llegar a tener aspectos o factores sociales en la permanencia de los estudiantes en los programas académicos matriculados de educación superior, no ha sido ajeno para otros investigadores, lo que ocurre es que han sido relevados o dejados en un segundo plano de importancia, debido a que por lo general las investigaciones que abordan este tema, identifican factores determinantes de la deserción como son los económicos, académicos familiares, ejemplo de ello es como Narváez \& Cervantes (2019), clasifican los factores de la deserción en cuatro categorías:

Individuales (edad, género, estado civil, posición dentro de los hermanos, entorno familiar, calamidad y problemas de salud, integración social, incompatibilidad horaria con actividades extra académicas, expectativas no satisfechas, embarazo), académicas (orientación profesional, tipo de colegio, rendimiento académico, calidad del programa, métodos de estudio, resultado en el examen de ingreso, insatisfacción con el programa u otros factores, número de materias), institucionales (normalidad académica, becas y formas de financiamiento, recursos universitarios, orden público, entorno político, nivel de interacción personal con los profesores y estudiantes, apoyo académico, apoyo psicológico) y socioeconómicas ( estrato, situación laboral, situación laboral de los padres e ingresos, dependencia económica, personas a cargo, nivel educativo de los padres, entorno macroeconómico del país).

Pero al comparar detalladamente las categorías planteadas por Narváez y Cervantes, con los resultados de la presente investigación, se encuentran coincidencias, debido a que al preguntarle a los estudiantes porque motivos han pensado en abandonar o suspender sus estudios, las respuestas se distribuyen así: Un $41 \%$ por motivos económicos, un $22 \%$ por aspectos laborales, un $11 \%$ por factores asociados a la academia, un $5 \%$ por aspectos de orden familiar, un $4 \%$ por cuestiones de salud, y un $17 \%$ de los estudiantes señalan otras causas. De esta manera se evidencia que los 
factores que propician la deserción son muy similares en las diferentes investigaciones realizadas.

Además, en algunas de dichas categorías, se incluyen aspectos sociales como la interacción entre profesores y estudiantes, pero no se identifican la importancia que pueden llegar a tener aspectos sencillos como el desarrollo de talentos que tengan los estudiantes, y que les pueden facilitar la vida universitaria, y más aún en educación a distancia, mediada a través de la virtualidad.

Por su parte Escañes, Herrero \& Merlino (2013), agrupan las variables que pueden incidir en la deserción en cinco conjuntos de temáticas: Individuales, socioeconómicas, académicos, institucionales y contextuales, que no distan de las identificadas por otros autores que abordan el tema de la deserción y permanencia. Pero llama la atención que en las temáticas académicas e institucionales, se tengan en común variables como el rendimiento académico y la satisfacción con la universidad, que en el caso de esta investigación el $59 \%$ de los estudiantes manifiestan tener un rendimiento académico alto, y un $22 \%$ sobresaliente, además el $47 \%$ y $41 \%$ señalan estar de acuerdo que la buena relación con la universidad nacional abierta y a distancia (UNAD) y sus servicios, han incidido en su permanencia en el programa matriculado. En este aparte es necesario señalar que la información primaria fue recolectada de estudiantes catalogados como antiguos, es decir del segundo al décimo periodo académico, que son los llamados a contar su experiencia en el desarrollo de sus programas.

Otro aspecto importante identificado en esta investigación fue el impacto que puede llegar a tener una adecuada relación entre docentes y estudiantes, donde un $88 \%$ la consideran totalmente indispensable para continuar sus estudios. Este factor también es considerado relevante en otras investigaciones, es el caso de Hamachek (1970, citado por Martínez \& Pérez, 2017) expresa: "Los maestros que demuestran un constante interés personal en el progreso de sus alumnos, tienen probabilidades de motivarlos más eficazmente que los maestros que se inclinan a adoptar una actitud distante e impersonal.", lo anterior muestra la importancia de la motivación que se puede ejercer por varias estrategias.

En este orden de ideas, Chalela, Valencia \& Arango (2017), señalan que:

Los factores motivacionales que llevan a un individuo a cursar un programa académico, es en primer lugar la calidad de este, y en el contexto colombiano los estudiantes buscan una formación que los prepare técnicamente para el mundo laboral, quieren que en el proceso se involucren conocimientos, infraestructura, política educativa, formación 
ciudadana, responsabilidad del estado y oportunidades de desarrollo individual.

Al respecto de este último factor motivacional, los estudiantes encuestados al señalar de manera puntual los talentos que poseen y que la universidad les puede ayudar a desarrollar, es una oportunidad para que los estudiantes obtengan un desarrollo individual complementario a la formación académica, dicho apoyo en el desarrollo de talentos, permite un acercamiento del estudiante con la institución, y más aún en educación virtual donde los estudiantes acuden poco a los centros de la universidad. Además, Rué (2016), dice que se deben generar mejores oportunidades formativas y de desarrollo, lo más relevantes posibles y para más ciudadanos.

En otras palabras, el aprendizaje para un futuro desconocido tiene que ser un aprendizaje orientado hacia el incremento y potenciación de las cualidades humanas y de las inclinaciones de los estudiantes. Es decir, adoptar dichas cualidades e inclinaciones como base de las propuestas formativas y trabajar desde las áreas de conocimiento en su máximo desarrollo. (Rué, 2016).

Así mismo, Altbach (2016), al explicar lo que debe ser la universidad, de tal manera que permita el desarrollo de los estudiantes en otros campos, señala lo siguiente: "la universidad moderna se encuentra en el centro de un sistema internacional de conocimientos que abarca la tecnología, las comunicaciones y la cultura. La universidad sigue siendo el centro primario de aprendizaje y el principal repositorio de sabiduría acumulada". En el caso de la cultura y la sabiduría acumulada encajan bien en este concepto moderno de universidad, debido a que los talentos de los estudiantes se pueden aprovechar para incrementar la cultura y por ende la sabiduría de la institución que beneficiara a todos los grupos interesados.

De esta manera y teniendo presente que la investigación original, busca la permanencia de los estudiantes matriculados en programas de la escuela ECACEN zona occidente, y que este working paper como producto de dicha investigación, pretende mostrar los aspectos o factores sociales, asimilados en este documento como talentos o hobbies que posean los estudiantes y que bien utilizados por parte de las instituciones, pueden llegar a fortalecer la vida universitaria y por ende propiciar la permanencia de los estudiantes, debido a que de acuerdo a Unamuno citado por Obarrio y Masferrer (2013), "la universidad es la llamada a generalizar el conocimiento, de los saberes, 
sin que ninguno de éstos pueda tener mayor importancia sobre otro, por lo que se ha de hallar un desarrollo equilibrado en todos los conocimientos".

Por su parte, León (2019), en su propuesta de plan estratégico de bienestar institucional para disminuir las cifras de deserción en la educación superior en Colombia, incluye entre las estrategias psicológicas que necesitan implementar las IES, y que propone el MEN para disminuir la deserción estudiantil, "la construcción de programas que fortalezcan las capacidades y recursos del estudiante en su proceso de formación humana. Además, señala que el bienestar educativo ofrece las garantías para el desarrollo social, cultural, lúdico, y cognoscitivo de profesores, estudiantes y trabajadores".

Por lo tanto, teniendo presente la información obtenida en la investigación y más precisamente en los aspectos de orden social que los estudiantes manifestaron poseer y desear que la universidad les ayude a potenciar, junto con lo propuesto por otros autores, en lo que tiene que ver con la parte que a las IES les corresponde, se puede inferir que no solo se puede enfrentar la deserción estudiantil, a través del abordaje de los factores económicos o académicos, si no que existen más opciones que bien aprovechados, contribuyen al mejor estar de los estudiantes en la institución, y propiciar una permanencia.

\section{CONCLUSIONES}

De acuerdo con los resultados obtenidos, se puede establecer que el factor económico tiene un peso muy importante al momento del estudiante tener que tomar la decisión de abandonar sus estudios, situación que es preocupante debido a que un buen porcentaje de los estudiantes financian sus estudios con recursos provenientes de una relación laboral.

De la misma manera, es necesario que las instituciones de educación superior y el ministerio de educación nacional, al diseñar e implementar los planes y estrategias tendientes a disminuir la deserción estudiantil, no solo busquen atacar los factores socioeconómicos, institucionales y familiares, si no que por el contrario construyan programas más robustos que aborden todos los factores que propician que se presente el fenómeno de la deserción.

Finalmente, se recomienda que las instituciones de educación superior, en este caso en particular la UNAD, identifiquen los talentos o hobbies que poseen los estudiantes, con la finalidad de potenciarlos en mejora del 
ambiente universitario, crear más sentido de pertinencia en los estudiantes y por ende contribuir a la permanencia estudiantil.

\section{REFERENCIAS}

Escanés, G., Herrero, V., \& Merlino, A. (2013). El Estudio De La Permanencia De Estudiantes en La Universidad: Elementos Para La Aplicación De Un Modelo De Duración. Cuadernos de Educación, 11(11), 1-14.

Cervantes Muñoz, M. A., \& Narváez Ferrín, M. E. (2019). Estudio prospectivo y estratégico para la gestión de la retención y la permanencia en el programa administración de empresas de la Universidad Nacional Abierta $Y$ A Distancia -UNAD. Documentos De Trabajo ECACEN, (2). doi: https://doi.org/10.22490/ECACEN.3026

Martínez Roa, D. A., \& Pérez Chacón, E. A. (2017). Factores motivacionales que influyen en la permanencia académica de los estudiantes del 4to año de la carrera de Psicología durante el 2do semestre 2016 en la UNAN Managua. (s.d.)

Chalela Naffah, S., Valencia Arias, A., \& Arango Botero, D. (2017). Motivaciones de los estudiantes universitarios para continuar con su formación académica en programas de posgrado. Revista Lasallista de Investigación, 14(2), 160-170. doi: https://doiorg.bibliotecavirtual.unad.edu.co/10.22507/rli.v14n2a15

Rué, J. (2016). Entornos de aprendizaje digitales y calidad en la educación superior. Barcelona, España: Editorial UOC. Recuperado de https://elibronet. bibliotecavirtual. unad.edu.co/es/ereader/unad/58440? page $=38$.

Gómez Lozano, M. T. \& Vinasco Castañeda, C. (Comp.). (2020). La voz del estudiante en la educación superior: un mundo por descubrir. Universidad de los Andes. Recuperado de https://elibronet.bibliotecavirtual.unad.edu.co/es/ereader/unad/127718?page $=79$

G. Altbach, P. (2016). Educación superior comparada: el conocimiento, la universidad y el desarrollo. Buenos Aires, Argentina: Editorial Nobuko. Recuperado de https://elibronet. bibliotecavirtual. unad.edu.co/es/ereader/unad/77185? page $=24$. 
팝 Escuela de Ciencias

Administrativas, Contables,

Económicas y de Negocios

Un:Nap

Obarrio, J. A., \& Masferrer, A. (2013). Expresión oral y proceso de aprendizaje: la importancia de la oratoria en el ámbito universitario.

Dykinson

León Fernández, S. M. (2019). Propuesta plan estratégico de bienestar institucional para disminuir las cifras de deserción estudiantil en la educación superior en Colombia para el año 2019. (s.d.)

Lerma, H. (2012). Metodología de la investigación: Propuesta, anteproyecto y proyecto. Bogotá, Colombia: ECOE EDICIONES. 